\title{
Mapeamento do Fluxo Geotérmico no Estado do Rio Grande do Sul
}

Antônio Jorge de Lima Gomes (antonio.gomes@ufvjm.edu.br), UFVJM; Jorge Luiz dos Santos Gomes (jorge.gomes@ufvjm.edu.br), UFVJM; e Valiya Mannathal Hamza (hamza@on.br), Observatório Nacional/MCT.

Copyright 2014, SBGf - Sociedade Brasileira de Geofísica

Este texto foi preparado para a apresentação no VI Simpósio Brasileiro de Geofísica, Porto Alegre, 14 a 16 de outubro de 2014. Seu conteúdo foi revisado pelo Comitê Técnico do VI SimBGf mas não necessariamente representa a pelo Coniâ da SBGf ou de deste material para propósitos comerciais sem prévia autorização da SBGt.

\section{Resumo}

Neste trabalho apresenta-se o mapeamento do fluxo geotérmico do Estado do Rio Grande do Sul, cujos dados foram compilados com base em levantamentos de estudos geotérmicos realizados a partir da década de 1970 e também em relatórios técnicos e publicações existentes na literatura. Os dados utilizados nesta compilação englobaram medidas experimentais em 58 localidades, sendo que 21 destes resultados estão situados dentro do estado nas regiões Norte, Nordeste, Noroeste e Centro do Rio Grande do Sul, e os restantes 37 dados estão situados no seu entorno. Os valores do fluxo geotérmico encontrado no Estado do Rio Grande do Sul estão compreendidos entre 30 e $121 \mathrm{~mW} / \mathrm{m}^{2}$ com um valor médio de $69 \pm 8 \mathrm{~mW} / \mathrm{m}^{2}$. Os altos valores situados na faixa de $100 \mathrm{~mW} / \mathrm{m}^{2}$ encontrados na borda da porção da Bacia do Paraná, dentro do Estado, é considerado como resultado de refração de calor dos sedimentos areníticos da bacia, no entanto a maior parte do estado apresentou fluxo geotérmico na faixa compreendida entre 48 e 72 $\mathrm{mW} / \mathrm{m}^{2}$, valores comuns de áreas continentais com coberturas sedimentares, consideradas tectonicamente estáveis.

\section{Introdução}

Mapear o fluxo geotérmico do Estado do Rio Grande do Sul e interpretar o seu campo térmico a nível regional, sobretudo na porção da Bacia do Paraná que está parcialmente situada dentro do Estado. Seus resultados vão contribuir para um maior entendimento do fluxo geotérmico terrestre a nível local, regional e global.

Este estudo faz parte de um projeto de pesquisa iniciado no ano de 2004 pelo Laboratório de Geotermia do Observatório Nacional - ON/MCT, cujo objetivo é a quantificação de recursos geotermais da Bacia do Paraná e tendo sua continuidade recíproca no Laboratório da UVJM - Universidade Federal dos Vales do Jequitinhonha e Mucuri, no Campus do Mucuri localizado em Teófilo Otoni.

Os primeiros mapeamentos geotérmicos em escala regional de gradiente e fluxo geotérmico no Brasil, e também na América do Sul, foram efetuados por Hamza e Muñoz (1996), Hurter e Pollack (1996), Hamza et al (2005) e Gomes e Hamza (2003, 2004, 2005, 2006, 2007, 2008, 2009) e Gomes (2009, 2012).

Grande parte da superfície do Estado do Rio Grande do Sul está contida dentro da Bacia do Paraná.

A Bacia do Paraná está situada na parte meridional do Brasil totalizando aproximadamente $1.400 .000 \mathrm{~km}^{2}$ de superfície, localizada em quatro países, com área de 1,1 milhões de $\mathrm{km}^{2}$ no Brasil, no Paraguai $100 \mathrm{mil} \mathrm{km}^{2}$,

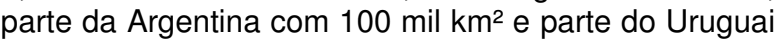
com 100 mil km².

\section{Metodologia}

Foram utilizados três métodos na determinação de gradientes geotérmicos e que foram respectivamente, 0 convencional (CVL), temperatura do fundo de poço de petróleo (BHT) e o de temperatura estável do fundo de poço (CBT). Todos os detalhes dessa metodologia foram apresentados em trabalhos anteriores de Hamza e Muñoz (1996), Gomes e Hamza (2004) e Hamza et al (2004 e 2005).

O método convencional (CVL) é utilizado para determinar o gradiente em intervalos de profundidades selecionados, com base em resultados de perfilagens em poços ou furos. Nos poços rasos com indícios de perturbações térmicas foram aplicadas correções para minimizar os efeitos de mudanças climáticas e de topografia local. Para um matriz de coeficientes $A M x N$, com $\mathrm{M}>\mathrm{N}$, e para um vetor $\hat{y}$ de dados observados 0 critério de mínimos quadrados fornece estimativas que satisfazem o modelo:

$$
\widehat{y}=A \hat{x}=A\left(A^{T} A\right)^{-1} A^{T} \bar{y}
$$

O método de temperatura do fundo de poço de petróleo (BHT) é utilizado somente em poços de petróleo e utilizam-se as temperaturas do fundo do poço e da média anual da superfície, que ajustadas em profundidade permitem a determinação do Gradiente. A relação utilizada para a determinação do gradiente geotérmico é:

$$
\Gamma=\left(T_{F P}-T_{0}\right) / H=\sum_{i=1}^{N}(d T / d z)_{i} h_{i} / \sum h_{i}
$$

onde TFP é a temperatura do fundo do poço, T0 a temperatura média anual da superfície, $\mathrm{H}$ a profundidade do poço, $\mathrm{N}$ o número de camadas e $\mathrm{h}$ a espessura da camada. As medições das temperaturas $\mathrm{BHT}$, efetuadas em poços de petróleo, precisam ser corrigidas dos efeitos perturbadores das atividades de perfuração. As medidas múltiplas de temperaturas, foram corrigidas pelos métodos propostos por Lachenbruch e Brewer (1959) e Middleton (1980). Nos casos em que havia apenas uma medida de temperatura utilizou-se a relação empírica da AAPG (1976).

O cálculo final do Fluxo é determinado pelo produto entre a condutividade térmica e o gradiente geotérmico do local, obtido pela seguinte relação:

$$
\mathrm{Q}=\mathrm{k} . \Gamma
$$

Onde: $Q$ é o fluxo geotérmico, $\mathrm{k}$ a condutividade térmica e $\Gamma$ o gradiente geotérmico. 


\section{Geologia e Geofísica regional}

Nas regiões Norte e Nordeste do Estado do Rio Grande do Sul está situada grande parte da Bacia do Paraná, fazendo parte do Planalto Meridional, formada por rochas vulcânicas mesozóicas. Na porção NE do Estado encontram-se as terras mais altas deste planalto com aproximadamente $1400 \mathrm{~m}$ de altitude.

De acordo com Kaul (1990) o estado do Rio Grande do Sul é constituído por terrenos rochosos cuja origem ou transformação retrocede aos mais diferentes períodos da história da crosta terrestre, trazendo o registro de distintos eventos geodinâmicos.

Segundo Almeida (1980) e Zalán et al. (1988 e 1990), constituem a Bacia do Paraná um pacote de rochas sedimentares e vulcânicas. As rochas ígneas do período Pré-Cambriano fazem parte do Escudo SulRio-Grandense e encontram-se muito erodidos. A altitude nessa região não ultrapassa os $600 \mathrm{~m}$.

A região central do Estado encontra-se a Cobertura de Sedimentos Cenozóicos, inseridos na Depressão Central, formando um corredor que corta toda a extensão $\mathrm{E}-\mathrm{W}$ da área, através de terrenos de baixa altitude. A Planície Costeira corresponde a uma faixa arenosa de $622 \mathrm{~km}$, com grande ocorrência de lagunas e lagoas.

\section{Resultados}

Apresenta-se na Figura (1) a localização dos 58 dados geotérmicos compilados dentro e fora do Estado do Rio Grande do Sul. Destes, temos dentro do Estado um total de 21 dados, 12 resultados são do método BHT, 8 dados são do CVL e apenas 1 dado é do método CBT.

Os dos demais valores do fluxo geotérmico do entorno do Estado constam da publicação de Gomes e Hamza (2008) e Gomes (2009).

Para um melhor entendimento do campo termal do Estado e suas inferências regionais, ainda, nesta compilação, apresentam-se os dados estudados em 21 locais no Estado e mais 37 dados em áreas vizinhas do Uruguai, Paraguai e Argentina, totalizando 58 localidades (Gomes e Hamza, 2008; Gomes, 2009).

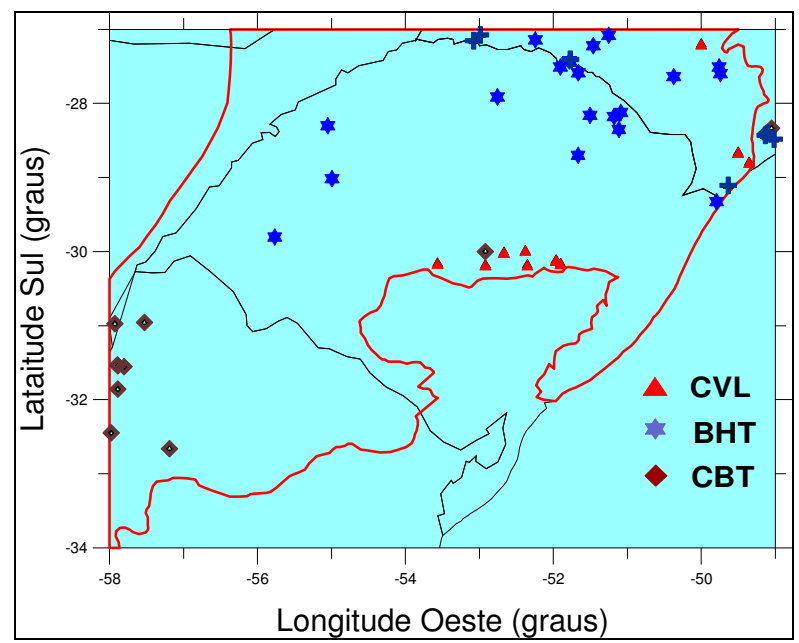

Figura 1 - Localização dos dados geotérmicos por tipo de gradiente no Estado e seu entorno.
Apresentam-se na Tabela (1) os valores do fluxo geotérmico do Estado e na Figura (2) a profundidade dos poços por tipo de gradiente geotérmico. Nas Figuras (3) e (4) apresenta-se o mapeamento do fluxo.

Tabela 1 - Fluxo Geotérmico por local e seu respectivo desvio padrão $(\sigma)$.

\begin{tabular}{|c|c|c|c|c|}
\hline \multirow{2}{*}{ Município } & \multicolumn{2}{|c|}{ Coordenadas } & \multicolumn{2}{c|}{$\mathbf{Q}\left(\mathbf{m W} / \mathbf{m}^{2}\right)$} \\
\cline { 2 - 5 } & Long. & Lat. & Médio & $\sigma$ \\
\hline \multicolumn{4}{|c|}{ Rio Grande do Sul } \\
\hline Alegrete & $-55,77$ & $-29,80$ & 44 & 3 \\
\hline Butiá & $-51,96$ & $-30,12$ & 74 & 3 \\
\hline Butiá & $-51,90$ & $-30,17$ & 100 & 4 \\
\hline Butiá & $-51,96$ & $-30,12$ & 101 & 4 \\
\hline Cachoeira do Sul & $-52,92$ & $-30,00$ & 52 & 2 \\
\hline Esmeralda & $-51,18$ & $-28,18$ & 49 & 2 \\
\hline Esmeralda & $-51,09$ & $-28,12$ & 49 & 2 \\
\hline Itacurubi & $-54,99$ & $-29,02$ & 48 & 2 \\
\hline Lagoa Vermelha & $-51,50$ & $-28,16$ & 48 & 2 \\
\hline Machadinho & $-51,66$ & $-27,59$ & 62 & 3 \\
\hline M. Ramos & $-51,90$ & $-27,51$ & 65 & 3 \\
\hline Muitos Capões & $-51,11$ & $-28,35$ & 55 & 2 \\
\hline Nova Bassano & $-51,66$ & $-28,70$ & 53 & 2 \\
\hline Paraíso do Sul & $-52,92$ & $-30,18$ & 64 & 3 \\
\hline Rio Pardo & $-52,38$ & $-29,99$ & 112 & 5 \\
\hline Rio Pardo & $-52,67$ & $-30,02$ & 109 & 5 \\
\hline Rio Pardo & $-52,35$ & $-30,18$ & 65 & 3 \\
\hline Ronda Alta & $-52,76$ & $-27,91$ & 52 & 3 \\
\hline São L. Gonzaga & $-55,05$ & $-28,30$ & 46 & 2 \\
\hline São Sepé & $-53,57$ & $-30,16$ & 122 & 5 \\
\hline Torres & $-49,79$ & $-29,33$ & 69 & 3 \\
\hline
\end{tabular}

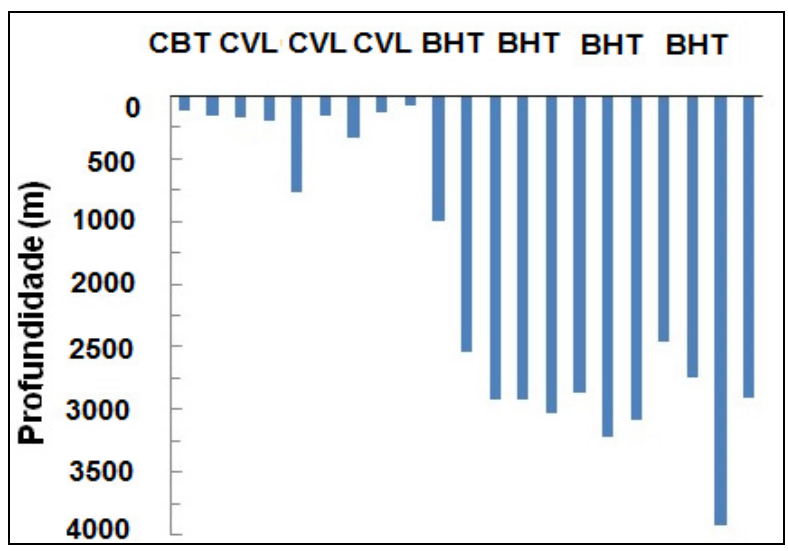

Figura 2 - Profundidade dos 21 poços localizados dentro do Estado do Rio Grande do Sul com seu respectivo tipo de gradiente geotérmico. 
Os resultados para o tipo $\mathrm{BHT}$, apresentaram valores de fluxo geotérmico compreendidos entre 44 e 70 $\mathrm{mW} / \mathrm{m}^{2}$.

Os resultados do tipo CVL estão compreendidos entre 64 e $121 \mathrm{~mW} / \mathrm{m}^{2}$.

A diferença entre os resultados CVL e BHT deve-se à presença dos basaltos que apresentam valores pequenos de condutividade térmica. Devido aos arenitos terem condutividade térmica muito superior aos basaltos, isto faz com que a camada basáltica se comporte como um isolante térmico, perturbando o valor da temperatura nas camadas rasas, resultando em maiores valores de gradiente e fluxo no método CVL em comparação com o tipo BHT.

Os valores do fluxo obtido com gradientes do tipo CBT estão compreendidos acima de $70 \mathrm{~mW} / \mathrm{m}^{2}$.

As regiões do Estado do Rio Grande do Sul situadas próxima à borda da Bacia do Paraná, apresentam valores de fluxo geotérmico compreendidos entre $60 \mathrm{e}$ $70 \mathrm{~mW} / \mathrm{m}^{2}$.

Constatamos que o comportamento térmico observado nas regiões da borda da Bacia do Paraná são de baixa entalpia, cujas águas quentes apresentam temperaturas na superfície de até $45^{\circ} \mathrm{C}$, resultando em gradientes médios de $30 \stackrel{\circ}{\circ} / \mathrm{km}$ e fluxo de $75 \mathrm{~mW} / \mathrm{m}^{2}$. Isto aponta que estes locais apresentam maior possibilidade de aproveitamento geotermal, tanto para atividades balneárias quanto para uso direto.

\section{Discussão e Conclusões}

Os valores obtidos para o fluxo geotérmico no Estado do Rio Grande do Sul estão compreendidos entre 30 e $121 \mathrm{~mW} / \mathrm{m}^{2} \mathrm{com}$ um valor médio de $69 \pm 8 \mathrm{~mW} / \mathrm{m}^{2}$.

Os resultados obtidos no mapeamento das figuras (3) e (4) indicam que os valores de fluxo geotérmico mais baixos, observados na parte interna da Bacia do Paraná, também são comuns nas áreas vizinhas, conforme é constatado no Estado de Santa Catarina e no país vizinho Uruguai, possivelmente ocasionado por movimento de fluídos nas formações areníticas da Bacia.

A região compreendida pela borda da interface de afloramento da Bacia da Bacia do Paraná dentro do Estado, apresenta os mais altos valores de fluxo geotérmico, respectivamente compreendidos entre $70 \mathrm{e}$ $121 \mathrm{~mW} / \mathrm{m}^{2}$. Estes valores estão situados na faixa de $100 \mathrm{~mW} / \mathrm{m}^{2}$ sendo considerados como resultado de refração de calor dos sedimentos areníticos vindos do interior da Bacia que estão por baixo da camada basáltica.

A maior parte do estado apresentou fluxo geotérmico na faixa compreendida entre 48 e $72 \mathrm{~mW} / \mathrm{m}^{2}$, valores normais em áreas continentais com coberturas sedimentares, consideradas tectonicamente estáveis.

Os poços profundos apresentam menor fluxo geotérmico e apontam para menor perturbação dos fluídos presentes nos arenitos e folhelhos da porção sedimentar da Bacia. Estudos avançados com mais detalhes poderão esclarecer melhor estas anomalias geotérmicas.

\section{Referências}

Eston, S.M., Hamza, V.M. e Becker, E.A., 1981, Pesquisas Geotérmicas na Exploração de Hidrocarbonetos na Bacia do Paraná, Relatório 16.379, Instituto de Pesquisas Tecnológicas do Estado de São Paulo-IPT.

Gomes, A.J.L. e Hamza, V.M., 2004. Mapeamento de Gradientes Geotérmicos no Estado de São Paulo, In: 1 Simpósio Regional de Geofísica, Anais, Cd-rom, São Paulo.

Gomes, A.J.L. and Hamza, V.M., 2005. Gradiente e Fluxo Geotérmico do Estado de Santa Catarina, 9응 International Congress of the Brazilian Geophysical, Salvador, Brasil.

Gomes, A.J.L. and Hamza, V.M., 2006. Gradiente e Fluxo Geotérmico no Estado do Paraná. In: II Simpósio de Geofísica da SBGf, 2006, Natal.

Gomes, A.J.L. and Hamza, V.M., 2007. Gradiente e Fluxo Geotérmico nas regiões Norte e Noroeste da Bacia do Paraná. In: 10 ICSBGf, Rio de Janeiro.

Gomes, A.J.L. and Hamza, V.M., 2008. Distribuição Regional de Gradiente e Fluxo Geotérmico no Estado do Rio Grande do Sul incluindo também áreas vizinhas do Uruguai e da Argentina. In: III Simpósio de Geofísica da SBGf, 2008, Belém.

Gomes, A.J.L. 2009, Avaliação de Recursos Geotermais da Bacia do Paraná, Tese de Doutorado, Observatório Nacional.

Hamza, V.M., Silva Dias, F.J.S., Gomes, A.J.L. and Terceros, Z.G.D., 2005. Numerical and Functional Representations of Regional Heat Flow in South America, Physics of the Earth and Planetary Interiors, Volume 152, 4, p.223-256.

Hamza, V.M. and Muñoz, M., 1996. Heat Flow map of South America, Geothermics, Vol. 25, nํ6, pp. 599646.

Hurter, S.J. and Pollack, H.N., 1996. Terrestrial heat flow in the Paraná basin, Southern Brazil, J. Geophysics.Res., 101, p.8659-8672.

Vitorello, I., Hamza, V.M.; Pollack, H.N. and Araújo, R.L.C., 1978. Geothermal Investigations in Brazil, Rev. Brasil. Geociências, vol. 8, pp. 71-89.

Vitorello, I., Hamza, V.M. and Pollack, H.N., 1980. Terrestrial Heat Flow in the Brazilian Highlands, Journal of Geophysical Research, vol. 85, pp. 37783788. 


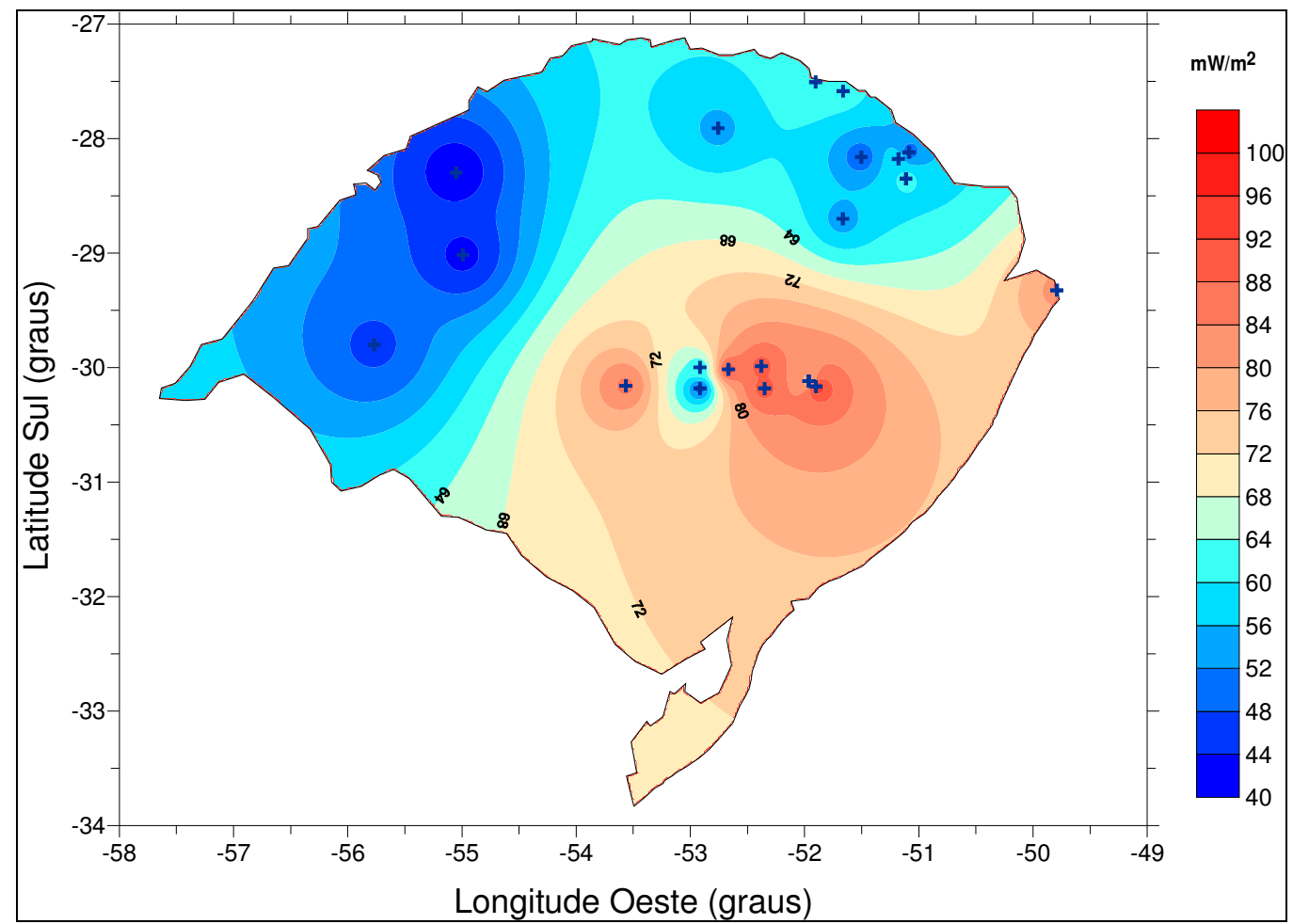

Figura 3 - Mapeamento de fluxo geotérmico regional considerando os dados dentro do Estado.

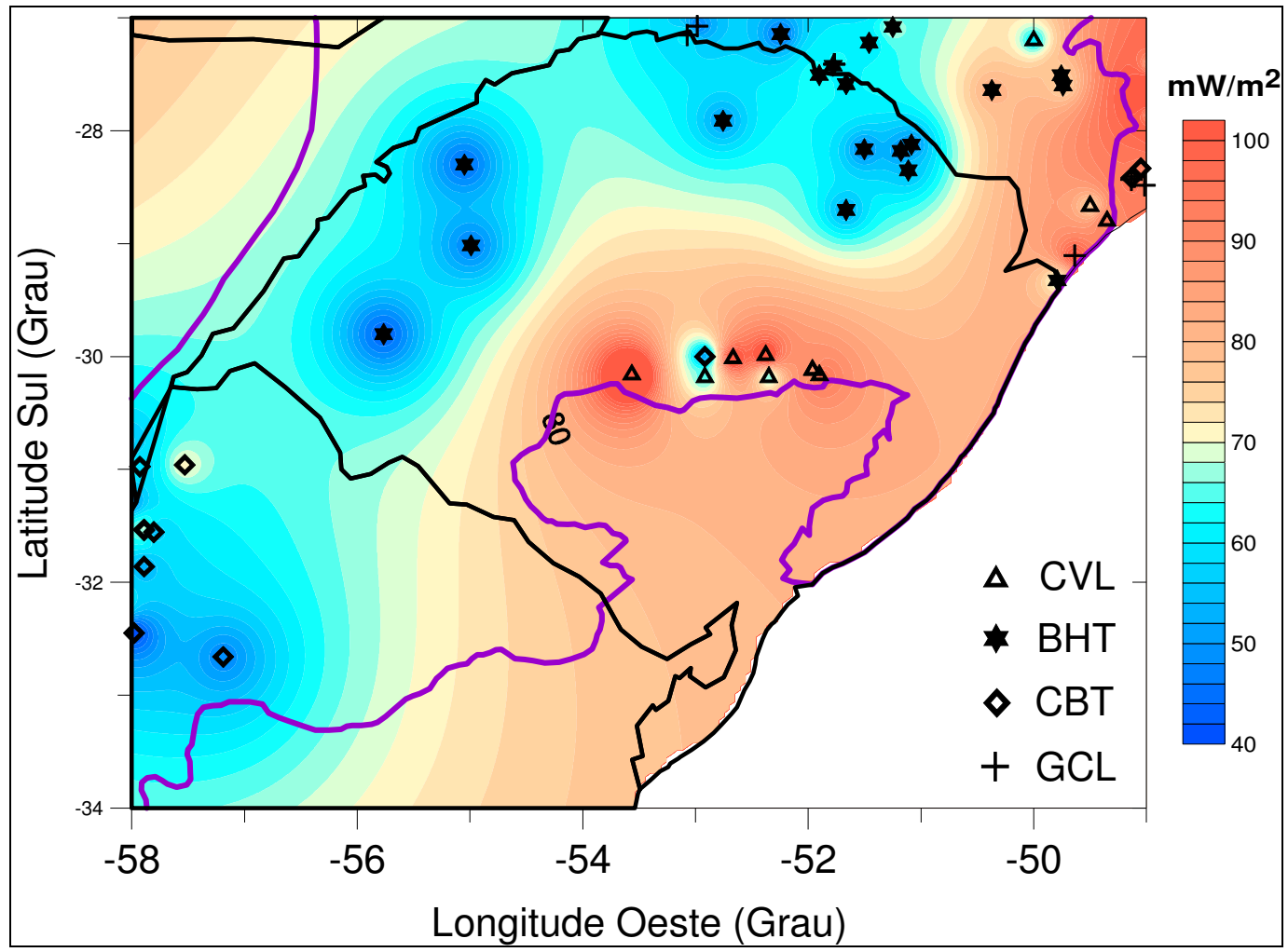

Figura 4 - Mapeamento de fluxo geotérmico regional com o contorno da Bacia do Paraná considerando os dados situados dentro e no entorno do Estado. 